\title{
Cosmic Microwave Background Anisotropies and Extra Dimensions in String Cosmology
}

\author{
A. Melchiorri, ${ }^{1,3}$ F. Vernizzi, ${ }^{1}$ R. Durrer, ${ }^{1}$ and G. Veneziano ${ }^{2}$ \\ ${ }^{1}$ Département de Physique Théorique, Université de Genève, 24 quai Ernest Ansermet, CH-1211 Genève 4, Switzerland \\ ${ }^{2}$ CERN Theory Division, CH-1211, Genève 23, Switzerland \\ ${ }^{3}$ Dipartimento di Fisica, Università di Roma Tor Vergata, Via della Ricerca Scientifica, Roma, I-00133, Italy
}

(Received 20 May 1999)

\begin{abstract}
A recently proposed mechanism for large-scale structure in string cosmology - based on massless axionic seeds - is further analyzed and extended to the acoustic-peak region. Existence, structure, and height of the peaks turn out to depend crucially on the overall evolution of extra dimensions during the pre-big bang phase: Conversely, precise cosmic microwave background anisotropy data in the acoustic-peak region will provide a window on the extra dimensions from string theory before their eventual compactification.
\end{abstract}

PACS numbers: 98.80.Cq, 98.70.Vc

One of the most stringent tests of inflationary cosmology will come when new precise satellite data on cosmic microwave background (CMB) anisotropies down to small angular scales will become available during the next few years [1]. Hopefully, these data will allow one not only to check whether the generic paradigm of inflation is valid, but also to make a strong selection among the multitude of models of inflation which are presently on the market. Models differ, in particular, on the presence or absence of a sizable tensor component (to be detected by polarization experiments), on the possible non-Gaussianity of the fluctuations (to be tested through higher-order correlations) and, finally, on the height and position of the socalled acoustic peaks in the multipole coefficients $C_{\ell}$ in the region $\ell>100$.

The pre-big bang (PBB) scenario [2], a particular model of inflation inspired by the duality properties of string theory, was thought for some time to be unable to provide a quasi-scale-invariant [Harrison-Zeldovich (HZ)] spectrum of perturbations. Indeed, first-order tensor and scalar perturbations were found to be characterized by extremely "blue" spectra [2]. The large tilt, together with a natural normalization imposed by the string cutoff at the shortest amplified scales $(\sim 1 \mathrm{~mm})$, makes their contribution to large-scale structure completely negligible.

It was later realized [3], however, that the spectral tilt of the supersymmetric partner of the dilaton, the universal axion of string theory (not to be confused with the PecceiQuinn axion), $\sigma$, can have a whole range of values, depending on the overall behavior of the six compactified internal dimensions. It is most useful to express the result in terms of the axion energy spectrum during the radiation era $[4,5]$. Let us define the tilt $\alpha$ by

$$
\Omega_{\sigma}(k, \eta) \equiv \rho_{c}^{-1} d \rho_{\sigma}(k, \eta) / d \log k \propto\left(k / k_{1}\right)^{\alpha},
$$

where, as usual, $\rho_{c}$ is the critical energy density, and $k_{1}$, related to the string scale, represents the end point of the spectrum. Assuming, as an example, separate isotropic behavior for the three external and the six internal dimensions, one finds

$$
\alpha=\frac{3+3 r^{2}-2 \sqrt{3+6 r^{2}}}{1+3 r^{2}},
$$

where $r \equiv \frac{1}{2}\left(\dot{V}_{6} V_{3}\right) /\left(V_{6} \dot{V}_{3}\right)$ is a measure of the relative evolution of the internal and external volumes.

Equation (2) allows for a range of values for the tilt $\alpha$. For static internal dimensions $(r=0)$ one finds a negative tilt, a "red" spectrum with $\alpha=3-2 \sqrt{3} \sim$ -0.46 ; for static external dimensions $(r=\infty)$ one finds a blue spectrum with $\alpha=1$ while, finally, for a globally isotropic evolution (modulo $T$ duality), i.e., for $r= \pm 1$, one obtains a flat $\mathrm{HZ}$ spectrum, $\alpha=0$ [4]. As we shall show in this paper, $\mathrm{CMB}$ anisotropy data prefer a slightly blue spectrum with $\alpha \sim 0.4$ leading to $r \sim 2.2$ so that the internal dimensions contract somewhat faster than the external dimensions expand. We note also that the pure power-law behavior in (2) is valid only if PBB evolution is not itself composed of various phases: It is conceivable, e.g., that some of the internal dimensions may "freeze" sometime during the PBB phase, in which case $\alpha$ will undergo a (negative) jump at some characteristic scale $k^{*}$ related to the freeze-out time. We will come to this possibility below.

The results of [3-5] reopened the possibility that PBB cosmology may contain a natural mechanism for generating large-scale anisotropy via the "seed" mechanism [6]. This possibility, which belongs to the generic class of isocurvature perturbations, is analyzed in [7] for massless axions, to which we limit our attention in this Letter, and in [8] for very light axions. Isocurvature perturbations from scalar fields have also been discussed in Ref. [9], but there the scalar field perturbations determine just the initial conditions. In our model the axion plays the role of a seed, as in scenarios with topological defects. The power spectrum of the seed is, however, not determined by causality, but the spectral index can vary (within the above limits). This reflects the fact that the axion field is generated during an inflationary phase. 
In the above papers a strong correlation between the tilt (the value of $n_{s}-1$ in standard notations) and normalization of the $C_{\ell}$ 's was noticed. A range of values around $n_{s}=1.2$ (slightly blue spectra) appeared to be favored by a simultaneous fit to the tilt and normalization on the large angular scales observed by Cosmic Background Explorer [10] to which the analysis in [7] was actually confined. In this paper we extend this study down to the small angular scales which have been explored observationally with limited precision so far [11] but which will become precisely determined during the next decade. We also supplement the analytic study of [7] with numerical calculations.

As in previous work $[7,8]$ we suppose that the contribution of the axions to the cosmic fluid can be neglected and that they interact with it only gravitationally. They then play the role of seeds which generate fluctuations in the cosmic fluid [6].

The evolution of axion perturbations is determined by the well-known axion-free background of string cosmology. One finds [7]

$$
\ddot{\psi}+\left(k^{2}-\frac{\ddot{a}_{A}}{a_{A}}\right) \psi=0,
$$

where we have introduced the "canonical" axion field $\psi=a_{A} \sigma$. The function $a_{A}=a e^{\phi / 2}$ is the axion pump field, $a$ denotes the scale factor in the string frame, and $\phi$ is the dilaton, which is supposed to be frozen after the pre-big bang/post-big bang transition. Dots denote derivation with respect to conformal time $\eta$. The initial condition for Eq. (3) is obtained from the pre-big bang solution and is then evolved numerically with $a_{A}=a$ during the post-big bang. The pre-big bang initial conditions require [7]

$$
\begin{gathered}
\sigma(\mathbf{k}, \eta)=\frac{c(\mathbf{k})}{a \sqrt{k}} \varphi(k, \eta), \quad \varphi(k, \eta)=\sin k \eta, \\
\eta \ll \eta_{\mathrm{eq}} .
\end{gathered}
$$

The deterministic variable $\varphi$ is a solution of Eq. (3), and $c(\mathbf{k})$ is a stochastic Gaussian field with power spectrum

$$
\left\langle|c(\mathbf{k})|^{2}\right\rangle=\left(k / k_{1}\right)^{-2|\mu|-1}=\left(k / k_{1}\right)^{\alpha-4},
$$

where we have related the tilt $\alpha$ introduced before to the parameter $|\mu|$ used in [7]. In order not to overproduce axions, we have to require $|\mu| \leq 3 / 2$, i.e., $\alpha \geq 0$. The limiting value $\alpha=0$ corresponds precisely to a $\mathrm{HZ}$ spectrum of CMB anisotropies on large scales [7].

The energy momentum tensor of the axionic seeds is given by

$$
T_{\mu}^{\nu}=\partial_{\mu} \sigma \partial^{\nu} \sigma-\frac{1}{2} \delta_{\mu}^{\nu}\left(\partial_{\alpha} \sigma\right)^{2} .
$$

Like $\sigma$ also the energy momentum tensor is a stochastic variable which is however not Gaussian. (The non-Gaussianity of the model has to be computed and compared with observations. But this is not the topic of the present work.)
For a universe with a given cosmic fluid, the linear perturbation equations in Fourier space are of the form

$$
\mathcal{D} X=S,
$$

where $X$ is a long vector containing all the fluid perturbation variables which depends on the wave number $\mathbf{k}$ and conformal time $\eta$. $S$ is a source vector which vanishes in the absence of seeds. $S$ consists of linear combinations of the seed energy momentum tensor and $\mathcal{D}$ is a linear ordinary differential operator. More concretely, we consider a universe consisting of cold dark matter, baryons, photons, and three types of massless neutrino with a total density parameter $\Omega=1$, with or without a cosmological constant $\left(\Omega_{\Lambda}=0.7\right.$ or 0.0$)$. We choose the baryonic density parameter $\Omega_{B}=0.05$ and the value of the Hubble parameter $H_{0}=100 h \mathrm{~km} \mathrm{~s}^{-1} \mathrm{Mpc}^{-1}$ with $h=0.5$. More details on the linear system of differential equations (7) can be found in Ref. [12], and references therein.

Since $S$ is a stochastic variable, so will be the solution $X\left(\eta_{0}\right)$ of Eq. (7). We want to determine power spectra or, more generally, quadratic expectation values of the form (with sums over repeated indices understood)

$$
\left\langle X_{i} X_{j}^{*}\right\rangle=\int_{\eta_{\text {in }}}^{\eta_{0}} G_{i l}(\eta) G_{j m}^{*}\left(\eta^{\prime}\right)\left\langle S_{l}(\eta) S_{m}^{*}\left(\eta^{\prime}\right)\right\rangle d \eta d \eta^{\prime},
$$

where $\mathcal{G}$ is a Green's function for $\mathcal{D}$.

We therefore have to compute the unequal time correlators $\left\langle S_{l}(\eta) S_{m}^{*}\left(\eta^{\prime}\right)\right\rangle$ of the seed energy momentum tensor. This problem can, in general, be solved by an eigenvector expansion method [13]. If the source evolution is linear, the problem becomes particularly simple. In this "coherent" case, we have

$$
S_{j}(\eta)=f_{j i}\left(\eta, \eta_{\text {in }}\right) S_{i}\left(\eta_{\text {in }}\right)
$$

with a deterministic transfer function $f_{i j}$. By a simple change of variables we can diagonalize the Hermitian, positive initial equal time correlation matrix, so that $\left\langle S_{l}\left(\eta_{\text {in }}\right) S_{m}^{*}\left(\eta_{\text {in }}\right)\right\rangle=\lambda_{l} \delta_{l m}$. Inserting this in Eq. (8) we obtain exactly the same result as by replacing the stochastic variable $S_{j}$ by the deterministic source term $S_{j}^{(\mathrm{det})}$ given by

$$
S_{j}^{(\mathrm{det})}(\eta) S_{i}^{(\mathrm{det}) *}\left(\eta^{\prime}\right)=\exp \left(\theta_{j i}\right) \sqrt{\left\langle\left|S_{j}(\eta)\right|^{2}\right\rangle\left\langle\left|S_{i}(\eta)\right|^{2}\right\rangle},
$$

where the phase $\theta_{j i}$ has to be determined case by case.

For our problem, the evolution of the pseudoscalar field $\sigma$ is linear, but the source, the energy momentum tensor of $\sigma$, is quadratic in the field. The same situation is met for the large- $N$ approximation of global $O(N)$ models. There one finds that the full incoherent result is not very different from the perfectly coherent approximation [12]. We hence are confident that we obtain relatively accurate results (to about 15\%) in the perfectly coherent approximation which we apply in our numerical calculation. A more thorough discussion of the accuracy of the coherent approximation will be given in a forthcoming 
paper [14]. Within the coherent approximation, we need just to determine the equal time correlators of the axion energy momentum tensor, $\left\langle T_{\mu \nu}(\mathbf{k}, \eta) T_{\rho \lambda}^{*}\left(\mathbf{k}^{\prime}, \eta\right)\right\rangle$, which are fourth order in $\sigma$. We then split the perturbations into scalar, vector, and tensor parts which completely decouple within linear perturbation theory. We determine the CMB anisotropies by numerically solving Eq. (3) and inserting the resulting source functions in a Boltzmann solver.

As discussed in [7], the amplitude of the CMB anisotropies depends on the small scale cutoff $k_{1}$ of the axion spectrum and the ratio between the string scale $M_{s}$ and the Planck mass $M_{P}$ in the way

$$
\ell(\ell+1) C_{\ell} \simeq\left(M_{s} / M_{P}\right)^{4}\left(\ell / k_{1} \eta_{\mathrm{dec}}\right)^{2 \alpha} .
$$

The simplest assumption, $k_{1} / a_{1} \sim M_{s} \simeq 10^{-2} M_{P} \simeq$ $10^{17} \mathrm{GeV}$, leads to the correct normalization only if $\alpha \lesssim 0.1$. Otherwise the tilt factor $\left(k_{1} \eta_{\mathrm{dec}}\right)^{-2 \alpha} \sim 10^{-60 \alpha}$ entirely suppresses fluctuations on large scales. The huge factor $k_{1} \eta_{\text {dec }}$ comes from extrapolating the spectrum over 30 orders of magnitude. If the tilt is larger than $\alpha \sim 0.1$, as suggested by the data (see below), we need either a slightly scale dependent tilt or some cutoff in the small scale fluctuations at later times. These possibilities are both physically plausible. The first one is realized if the compactified dimensions evolve more rapidly at the beginning of the dilaton-driven inflationary phase than towards its end. In other words, the parameters $r$ and $\alpha$ in Eq. (2), instead of being constant, will be a (slowly) decreasing function of time. One could thus have a rather blue spectrum at large scales, as necessary in order to have pronounced peaks, and a much flatter spectrum at small scales which helps avoiding normalization problems. We explore these questions in more detail in a forthcoming paper [14].

In Fig. 1 we show the scalar, vector, and tensor contributions to the resulting CMB anisotropies for an axion spectrum with tilt $\alpha=0.1$. The "hump" at $\ell \sim 40$ is due to the isocurvature nature of the perturbations. They are also the main reason why the acoustic peaks are very low. The result is remarkably similar to the large- $N$ case studied in Ref. [12]. The main difference here is that, as for usual inflationary models, we dispose of a spectral index which is basically free. By choosing slightly bluer spectra, we enhance the power on smaller scales.

In Fig. 2 we compare the results from different tilts with and without cosmological constant. The CMB power spectra obtained can have considerable acoustic peaks at $\ell \sim 250-300$. Increasing the tilt $\alpha$ raises the acoustic peaks and moves them slightly to smaller scales. As found in Ref. [7], the power spectrum of the scalar component is always blue. The tensor and vector components counterbalance the increase of the tilt, maintaining a nearly scale invariant spectrum on large scales. The models can be discriminated from the common inflationary spectra by their isocurvature hump and by the position of the first peak. We have compared our results with the latest experiments [11]. All the models agree quite well with the large-scale

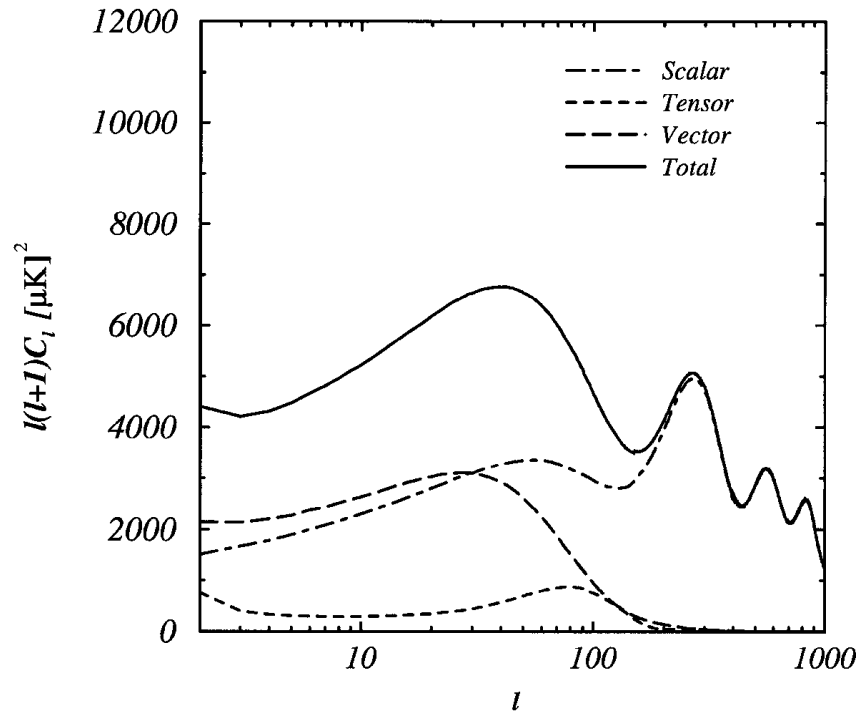

FIG. 1. The CMB anisotropy power spectrum for fluctuations induced from axionic seeds with a tilt $\alpha=0.1$. We show the scalar (dot-dashed line), vector (dashed line), and tensor (dotted line) contributions separately as well as their sum (solid line).

experiments, while on degree and subdegree scales, models with $0.3 \lessgtr \alpha \lesssim 0.5$ are favored by the data as can be seen from the $\chi^{2}$ analysis presented in Table I. For comparison, the $\chi^{2}$ of a standard $\Lambda$-CDM model, with theoretical errors given by cosmic variance, is 120 . However, we have to be aware that the $\chi^{2}$ test with present observations is a very rough indication of the goodness of a model, since the $C_{\ell}$ 's do not obey a Gaussian distribution [15]. This is especially serious for experiments with low sky coverage.

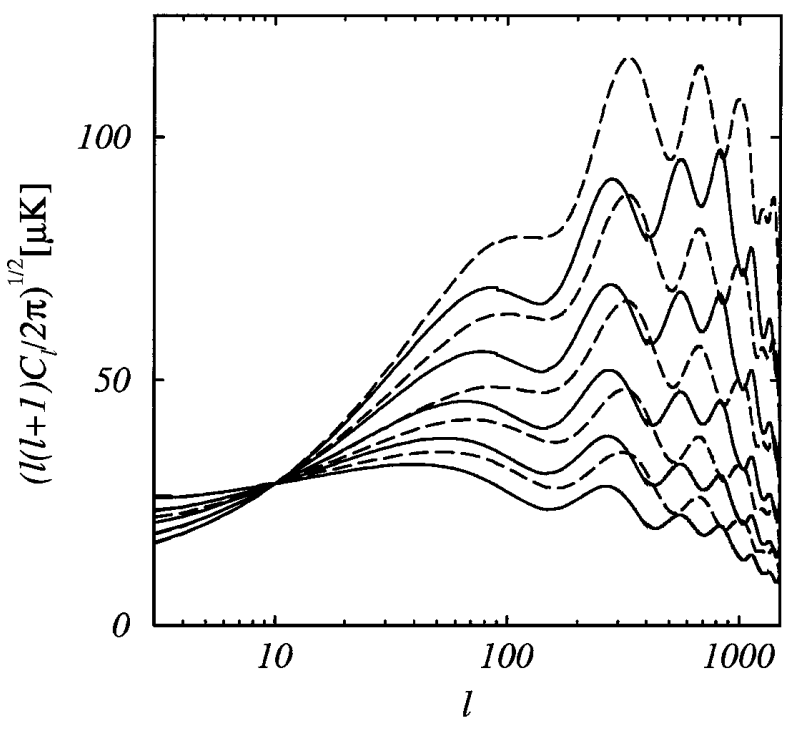

FIG. 2. The CMB anisotropy power spectrum for fluctuations induced by axionic seeds. We show the sum of the scalar, vector, and tensor contributions for five different tilts, with $\Omega_{\Lambda}=0$ (solid line) and $\Omega_{\Lambda}=0.7$ (long-dashed line). The tilt is increasing from bottom to top, $\alpha=0.1,0.2,0.3,0.4,0.5$. 
TABLE I. The value of $\chi^{2}$ (with $15 \%$ theoretical errors) from all the CMB anisotropy experiments compiled in Ref. [11] are presented for all the models. We compare with $N=60$ data points. Clearly, $\alpha \sim 0.4$ with $\Lambda=0$ or 0.7 is a reasonable fit to the data.

\begin{tabular}{lccccr}
\hline \hline & & \multicolumn{3}{c}{$\alpha$} \\
& 0.1 & 0.2 & 0.3 & 0.4 & 0.5 \\
\hline$\chi^{2}$ for $\Lambda=0$ & 302 & 214 & 119 & 66 & 82 \\
$\chi^{2}$ for $\Lambda=0.7$ & 249 & 152 & 111 & 70 & 119 \\
\hline \hline
\end{tabular}

In Fig. 3, the theoretical dark matter power spectra are compared with the data as compiled by Peacock and Dodds [16]. Models without a cosmological constant disagree in shape and amplitude with the data. The root mean square mass fluctuation within a ball of radius $8 h^{-1} \mathrm{Mpc}$ for these models is $\sigma_{8}=0.36,0.56,0.88,1.36,2.05$ for the tilts from $\alpha=0.1$ to $\alpha=0.5$, respectively. Models with a cosmological constant are in reasonable agreement with the shape of the spectrum (see Fig. 3). The values of $\sigma_{8}$ for these models are $0.21,0.38,0.53,0.82,1.25$, respectively. We estimate a (normalization) error of up to $\sim 30 \%$ in these numbers, due to the perfectly coherent approximation. Analysis of the abundance of galaxy clusters suggest $\sigma_{8} \sim 0.5\left(1-\Omega_{\Lambda}\right)^{-0.5}$ [17]. Since we can choose a blue, tilted spectrum in our model, we have more power on small scales and are able to fit large-scale structure data much better than defect models for which the spectral index is fixed by causality.

In this Letter we have presented preliminary results for the CMB anisotropies and linear matter power spectra in a pre-big bang scenario with axionic seeds. Because of

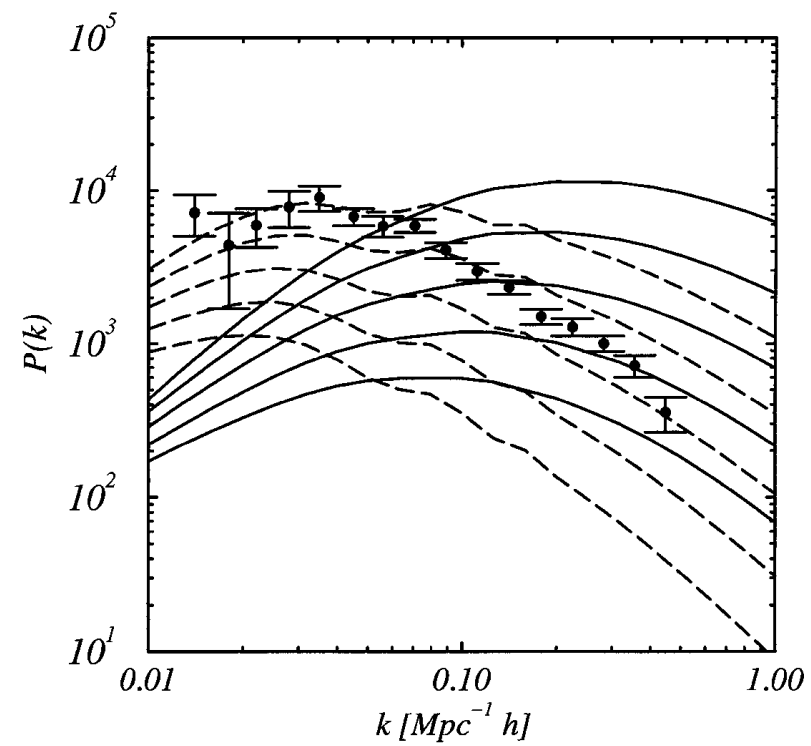

FIG. 3. The linear dark matter power spectrum for fluctuations induced by axionic seeds is compared with data for same values of the tilt as in Fig. 2. The spectra for the $\Omega_{\Lambda} \neq 0$ models (dashed lines) are shown with a bias factor of $b=1.2$. The value of the tilt increases from bottom to top as in Fig. 2. the isocurvature nature of the perturbations, a positive tilt $0.3 \lesssim \alpha \lesssim 0.5$ is required to fit the measured CMB anisotropy. Including a cosmological constant of $\Omega_{\Lambda} \sim$ 0.7 , as suggested by the recent supernovae results [18], the matter power spectrum is also in good agreement with measurements.

If improved data confirm the need of a significant tilt, $\alpha>0.1$, the most simple scenario $\left(k_{1} / a_{1}=M_{s}\right.$ and $\alpha=$ const) will be ruled out. This shows that CMB anisotropies may contain information about the evolution of extra dimensions. But clearly, also in this case the model remains highly predictive. It is easily distinguished from the more standard adiabatic models by its "isocurvature hump" at $\ell<100$ and the position of the first acoustic peak at $\ell \sim 300$. These values depend only slightly on the tilt (see Fig. 2). Furthermore the ratios between the scalar, vector, and tensor contributions are entirely fixed by the model.

We are grateful to Ram Brustein for helpful comments and to Nicola Vittorio for his Boltzmann code. This work is supported by the Swiss NSF.

[1] See the web sites: http://map.gsfc.nasa.gov and http:// astro.estec.esa.nl/SA-general/Projects/Planck

[2] G. Veneziano, Phys. Lett. B 265, 287 (1991); M. Gasperini and G. Veneziano, Astropart. Phys. 1, 317 (1993). An updated collection of papers is available at http://www.to.infn.it/ gasperin.

[3] E. J. Copeland, R. Easther, and D. Wands, Phys. Rev. D 56, 874 (1997).

[4] A. Buonanno et al., J. High Energy Phys. 01, 004 (1998).

[5] R. Brustein and M. Hadad, Phys. Rev. D 57, 725 (1998).

[6] R. Durrer, Phys. Rev. D 42, 2533 (1990); Fundam. Cosm. Phys. 15, 209 (1994).

[7] R. Durrer, M. Gasperini, M. Sakellariadou, and G. Veneziano, Phys. Rev. D 59, 043511 (1999); Phys. Lett. B 436, 66 (1998).

[8] M. Gasperini and G. Veneziano, Phys. Rev. D 59, 043503 (1999).

[9] P. J.E. Peebles, Astrophys. J. 510, 523 (1999); 510, 531 (1999).

[10] G.F. Smoot et al., Astrophys. J. 396, L1 (1992); C. L. Bennett et al., Astrophys. J. 430, 423 (1994).

[11] M. Tegmark, http://www.sns.ias.edu/ max; A. D. Miller et al., e-print astro-ph/9906421.

[12] R. Durrer, M. Kunz, and A. Melchiorri, Phys. Rev. D 59, 123005 (1999).

[13] N. Turok, Phys. Rev. D 54, 3686 (1996).

[14] F. Vernizzi et al. (to be published).

[15] J. R. Bond, A. H. Jaffe, and L. Knox, astro-ph/9808264, Astrophys. J. (to be published).

[16] J. Peacock and S. Dodds, Mon. Not. R. Astron. Soc. 267, 1020 (1994).

[17] V. Eke, S. Cole, and C. Frenk, Mon. Not. R. Astron. Soc. 282, 263 (1996).

[18] S. Perlmutter et al., Astrophys. J. (to be published). 